\title{
Ti-6Al-2Sn-2Zr-2Mo-2Cr Alloy for High Strength Aerospace Fasteners
}

\author{
Sesh A. Tamirisakandala ${ }^{1}$, Manish $\mathrm{Kamal}^{2}$ \\ ARCONIC Engineered Structures, 1000 Warren Avenue, Niles OH 44446, USA \\ ARCONIC Fastening Systems, 900 Watson Center Road, Carson, CA 90745 USA \\ Sesh.Tamirisakandala@Arconic.com
}

\begin{abstract}
$\underline{\text { Abstract }}$
Next generation demanding aerospace systems requirements are pushing the titanium alloy performance needs beyond the upper limits of the workhorse alloy $\mathrm{Ti} 6 \mathrm{Al}-4 \mathrm{~V}$ ( $\mathrm{Ti}$ 6-4), necessitating the use of advanced solutions. This paper provides an overview of Arconic's lightweight solution to address the needs of future aerospace fastening systems. The key attributes for aerospace fasteners are strength (tensile, double shear, and fatigue) and manufacturability (ability to forge heads and roll threads while meeting metallurgical and dimensional requirements) at an affordable cost. In particular, increasing double shear strength (DSS) while meeting other requirements is very challenging. Typically, DSS is about $60 \%$ of the tensile strength for Ti $6-4$, restricting Ti applications to moderate strength levels. Limited deep hardenability of Ti $6-4(\leq 0.5$ ") also restricts the usage to smaller diameter fasteners. Beta Ti alloys (e.g. Beta C) capable of achieving high tensile strengths suffer from shortfalls in DSS and producibility. There is a need for an affordable high strength Ti alloy that can extend titanium fastener usage to higher strength levels and larger size (up to 1"), which will enable reduction in number of joints and weight reductions by replacing higher density nickel/steel fasteners. Ti 6Al-2Sn-2Zr-2Cr-2Mo (Ti 6-22-22), a judiciously balanced $\alpha+\beta$ Ti alloy, designed and developed by RMI Titanium Company in the early 1970 s for thick-section aerospace structural applications with a need for higher strengths than Ti 6-4, is capable of meeting demanding fastener requirements of next generation aerospace systems. Superior producibility and ability to tailor processing-microstructure-property relationships in Ti 6-22-22 for achieving performance improvements will be discussed in this paper.
\end{abstract}

\section{Introduction}

Fasteners are critical components of aerospace systems and their selection is driven by a complex set of specifications and probability of failure models. Fastener material selection is often made based on prior experience, cost, and availability considerations. Higher performance demands, corrosion issues associated with dissimilar materials joints, and overall system-level weight reduction goals of the next generation aircrafts are challenging fastener choices. The key attributes for aerospace fasteners are strength (tensile, double shear, and fatigue) and manufacturability (ability to forge heads and roll threads while meeting metallurgical and dimensional requirements) at an affordable cost. Double shear strength (DSS) is a particularly challenging property to increase while meeting other requirements [1]. Typically, DSS is about $60 \%$ of the tensile strength for Ti-6-4, restricting Ti applications to moderate strength levels. Limited deep hardenability of Ti-6-4 ( $\leq 0.5$ ") also restricts the usage to smaller diameters. Beta Ti alloys (e.g. Beta C) capable of achieving high tensile strengths suffer from shortfalls in DSS and producibility. There is a need for an affordable high strength $\mathrm{Ti}$ alloy that can extend titanium fastener usage to higher strength range and larger sizes up to 1", which will enable reduction in number of joints and weight reductions by replacing higher density nickel/steel fasteners. An affordable titanium alloy fastener class with a target tensile strength of $180 \mathrm{ksi}$ and DSS of $105 \mathrm{ksi}$ up to 1 " diameter provides a potential of up to $30 \%$ weight reduction by replacing heavier nickel and or corrosion resistant steel fasteners (Figure 1) and reducing the number of joints. Ti 6Al-2Sn-2Zr-2Mo-2Cr-0.15Si (Ti 6-22-22 or Ti6Q2), a potential Ti alloy capable of meeting these targets, is the focus of this paper.
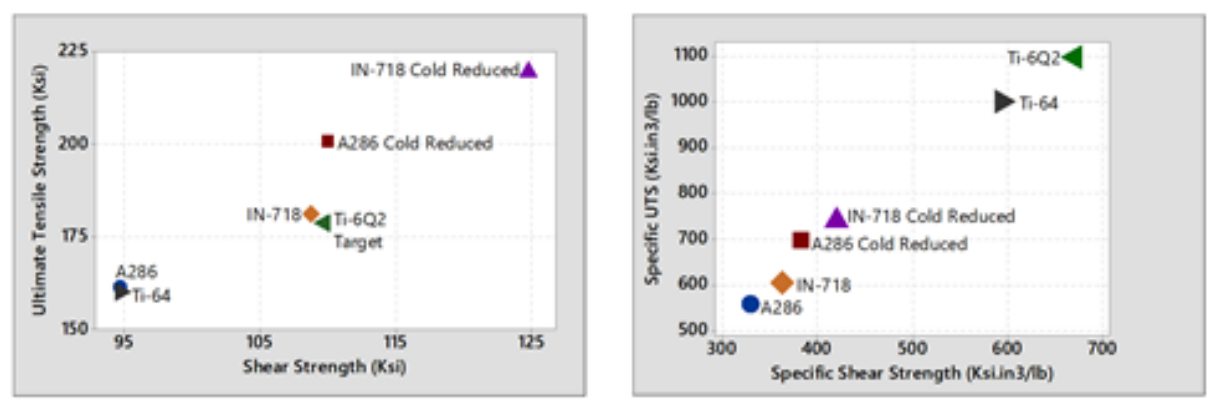

Figure 1: Comparison of tensile strength (left) and specific tensile strength (right) of various aerospace fastener classes.

\section{Background on Ti 6-22-22 Alloy}

Ti 6-22-22 (all compositions expressed in weight percent) is an $\alpha+\beta$ alloy developed and specified for thick section (up to 4"), structural airframe applications that require higher strengths than Ti 6Al-4V (Ti 6-4) in combination with high damage tolerance 
without significant density $(\rho)$ penalty $\left(\rho_{\mathrm{Ti}} 6-22-22=0.164 \mathrm{lb} / \mathrm{in}^{3}\right.$ vs. $\left.\rho_{\mathrm{Ti}} 6-4=0.160 \mathrm{lb} / \mathrm{in}^{3}\right)$. The Ti 6-22-22 alloy was originally formulated by RMI Titanium Company in the early 1970s [2] for elevated temperature applications in gas turbine engines, but the alloy was not competitive for engine applications compared to metastable $\beta$ alloys Ti 6Al-2Sn-4Zr-6Mo (Ti 6-2-4-6) and Ti 5Al$2 \mathrm{Sn}-2 \mathrm{Zr}-4 \mathrm{Mo}-4 \mathrm{Cr}$ (Ti-17) developed around the same timeframe. Melting, processing, and specifications development were subsequently resumed in 1991 by RMI and Lockheed Martin when the alloy was determined to have potential for thick section, F22 structural airframe applications [3]. Ti 6-22-22 has demonstrated the potential for significant performance enhancements in thick-section components due to improved strength, elastic modulus, and weight reduction concurrent with excellent damage tolerance properties compared to the workhorse Ti 6-4, which is limited to 1-inch or less sections. Alloy formulation of Ti 6-22-22 was judiciously balanced to increase strength while maintaining high damage tolerance in thicker sections. In addition to Al, solid solution strengthening in Ti 6-22-22 is achieved via additions of neutral elements Sn and Zr. Hardenability of the alloy was increased through additions of $\beta$ phase stabilizers Mo (isomorphous element) and $\mathrm{Cr}$ (eutectoid element). The comparatively higher concentration of $\beta$ stabilizing elements Mo and $\mathrm{Cr}$ render Ti 6-22-22 deep hardenable in thick-section forgings and plate cooled from solution temperatures at fan air cooling rates. Oxygen content in Ti 6-22-22 was kept below 0.13\% (extra-low interstitial ELI grade) for maximizing fracture toughness. Silicon up to $0.15 \%$ was added with an intention to increase room and elevated temperature strength, modulus, improve thermal stability, and creep resistance. Early work on Ti 6-22-22 led to the development of the triplex heat treatment schedule to obtain an optimized combination of strength, toughness, and fatigue properties necessary for high durability and damage tolerance thick-section plate and forged components for service in fracture critical applications. Triplex heat treatment comprises of: i) $\beta$ solution treatment, ii) $\alpha+\beta$ solution treatment, and iii) aging. The $\beta$ solution treat step creates fully lamellar/basketweave microstructure in prior $\beta$ grains for maximizing fracture toughness, $\alpha+\beta$ solution step coarsens $\alpha$ plates improving microcrack propagation resistance (i.e. slower fatigue crack growth rate), and final aging precipitates fine secondary $\alpha$ platelets from metastable $\beta$ retained in the $\beta$ ribs providing an increase the yield strength. Despite the presence of grain boundary $\alpha$, triplex heat treated microstructure supports an improved balance of strength and toughness properties compared to solution treated and aged Ti 6-4. This Ti 6-22-22 microstructure also supports fatigue crack growth rate properties that are comparable to $\beta$ annealed Ti 6-4. Damage tolerant components machined out of closed-die forgings or plate up to 4 " section thickness are currently flying on F-22 and F-35 fighter aircrafts for over 20 years.

Duplex microstructure variant (primary $\alpha$ particles/grains) plus secondary $\alpha$ platelets in equilibrium $\beta$ matrix) of Ti 6-22-22 produced via $\alpha+\beta$ solution treat plus age (STA) after $\alpha+\beta$ forging (Figure 2) would be of interest for high strength and moderate toughness applications. Compared to metastable $\beta$ alloys such as Ti 5Al-5V-5Mo-3Cr (Ti 5-5-5-3), Ti 6-22-22S, an $\alpha+\beta$ alloy, offers advantages of lower density (i.e. high specific strength), improved microstructural stability, higher temperature capability, and lower alloy formulation cost. Conceptually, duplex microstructure generated by $\alpha+\beta$ STA can fully exploit the strength increase via precipitation strengthening mechanism of fine secondary $\alpha$ platelets provided the cooling rate from $\alpha+\beta$ solution temperature is rapid enough to retain metastable $\beta$ phase [4]. Primary $\alpha$ characteristics are dictated by solution temperature and cooling rate. Solute partitioning occurs during cooling from solution temperature if there is sufficient time for diffusion. Primary $\alpha$ will be rich in $\mathrm{Al}$ and $\mathrm{O}$ (promoting $\mathrm{Ti}_{3} \mathrm{Al}$ formation) and $\alpha$ lamellae will be lean in $\mathrm{Al}$ and $\mathrm{O}$. The $\alpha$ colony size is approximately equal to $\beta$ grain size at the solution temperature. With decrease in cooling rate from surface to center, volume fraction of metastable $\beta$ reduces and volume fraction of coarse $\alpha$ plates formed by nucleation and growth increases. The coarse $\alpha$ plates contribute less to the yield stress because they are large enough to deform plastically and only $\alpha / \beta$ boundary hardening is present as a hardening mechanism (plus a small contribution from dislocation hardening due to increased dislocation density in the $\beta$ matrix during $\alpha$ precipitation). Homogeneous precipitation of fine incoherent platelets of $\alpha$ from metastable $\beta$ occurs when aged at high temperature for sufficient time. Secondary $\alpha$ platelets (typically $<0.5 \mu \mathrm{m}$ wide and aspect ratio in the range $5-10$ ) obey burgers orientation relationships (BORs) with the flat surface parallel to one of $\{112\}_{\beta}$. Only two or three variants of BORs (out of 12 possibilities) being nearly perpendicular to each other dominate in a given volume of a $\beta$ grain to minimize overall elastic strains. Incoherent secondary $\alpha$ platelets are too small to deform plastically. Hard, undeformable secondary $\alpha$ platelets produce significant increase in yield strength proportional to the inverse of inter-particle spacing via Orowan bowing of dislocations (bypass) mechanism. Aging time and temperature parameters control the size, spacing, and volume fraction of secondary $\alpha$ platelets that precipitate within retained $\beta$ (i.e. equilibrium $\beta$ ). Highest yield strength contribution is obtained when equilibrium volume fraction of secondary $\alpha$ platelets is present. The higher the aging temperature, the lower will be the equilibrium volume fraction of platelets but the aging time to reach maximum possible strength is shortened.

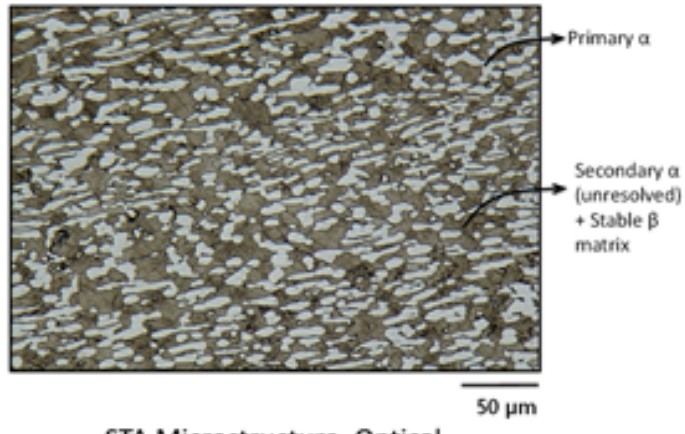

STA Microstructure, Optical

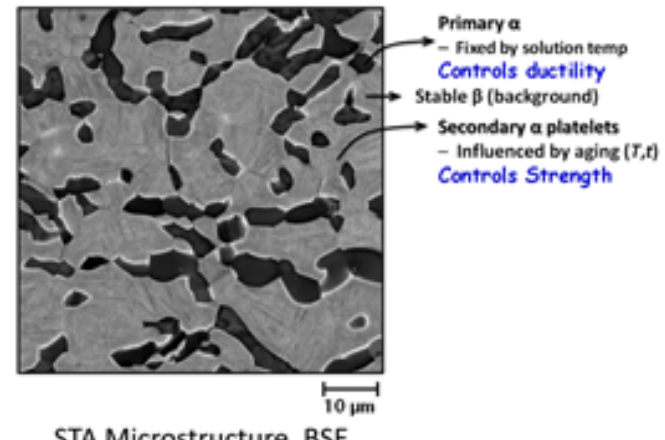

STA Microstructure, BSE

Figure 2: Typical microstructure of Ti-6-22-22 $\alpha+\beta$ STA condition, optical micrograph (left), backscattered electron (BSE) micrograph (right). 


\section{Ti 6-22-22 Fastener Product Development}

Bar stock of three different sizes $(0.25,0.5$, and 1" diameter) were produced using production Ti 6-22-22 material and equipment. A design of experiments (DOE) study was conducted using different bar size, solution treatment, and aging parameter combinations to establish the range of UTS, tensile elongation, and DSS combinations obtainable in Ti 6-22-22 and a summary of the DOE is presented in Figure 3. Optimum STA heat treat conditions for fasteners were downselected based on manufacturability, quality control, and performance requirements.
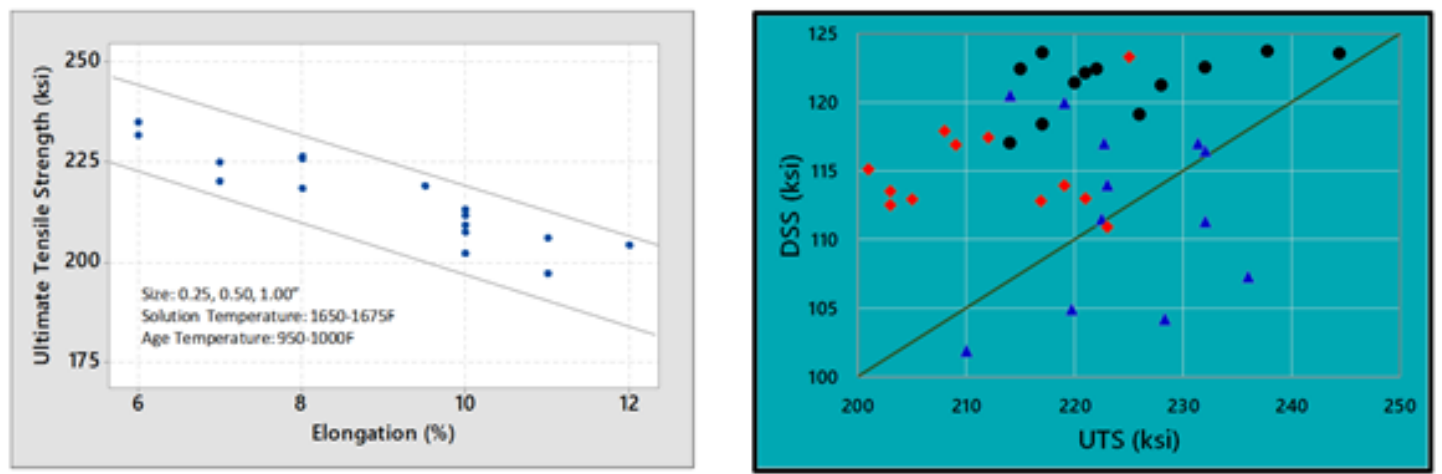

Figure 3: Variation of beta volume fraction with temperature (left) and tensile strength-elongation combinations in Ti 6-22-22 bar stock for various STA heat treatment conditions (right).

Fasteners of three different sizes were manufactured using production equipment and processes. Bolts after heading operation are shown in Figure 4 and typical microstructures in the head and shank regions are presented in Figure 5. Dimensional and metallurgical characteristics evaluations on fully finished 0.25 " diameter fasteners are presented in Figure 6 . Tensile and double shear test data generated on Ti 6-22-22 fasteners is compared with that of Ti 6-4 and IN-718, showing $20 \%$ improvement over Ti 6-4 and ability to meet IN-718 minimum requirements. Fasteners were also tested in tension-tension fatigue per MIL-STD-131211 at a stress ratio of 0.1 and lives higher than 60,000 cycles without failure were recorded.

\section{Summary and Conclusions}

Next generation aerospace systems significantly benefit from advanced high strength titanium fasteners. Ti-6Al-2Sn-2Zr- $2 \mathrm{Mo}-2 \mathrm{Cr}$ (Ti 6-22-22) is a judiciously balanced and fully matured alloy capable of replacing high-density nickel superalloy IN-718. Product development and testing efforts in this study demonstrated that Ti 6-22-22 meets critical requirements for high strength aerospace fasteners.

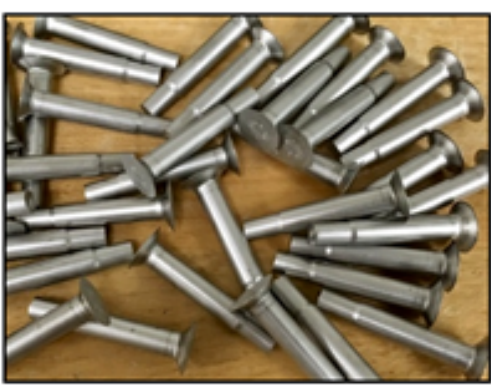

$0.25 " \varnothing$

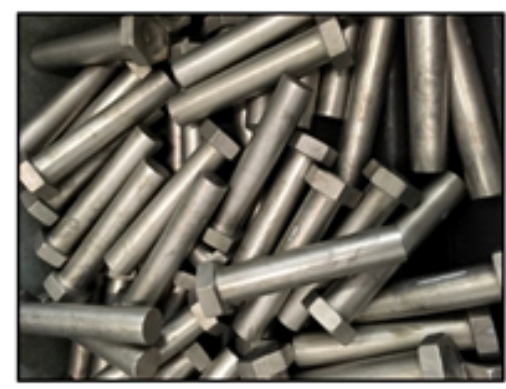

$0.5 " \varnothing$

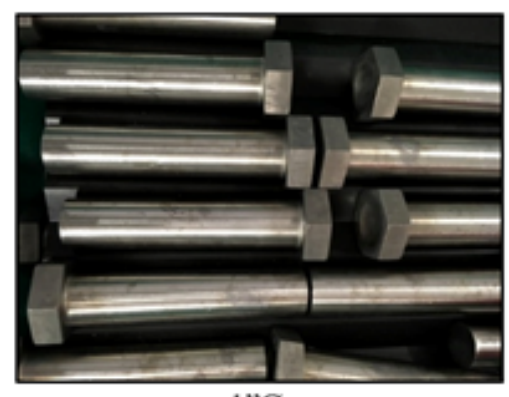

$1 " \varnothing$

Figure 4: Headed bolts of Ti 6-22-22 in three different sizes. 

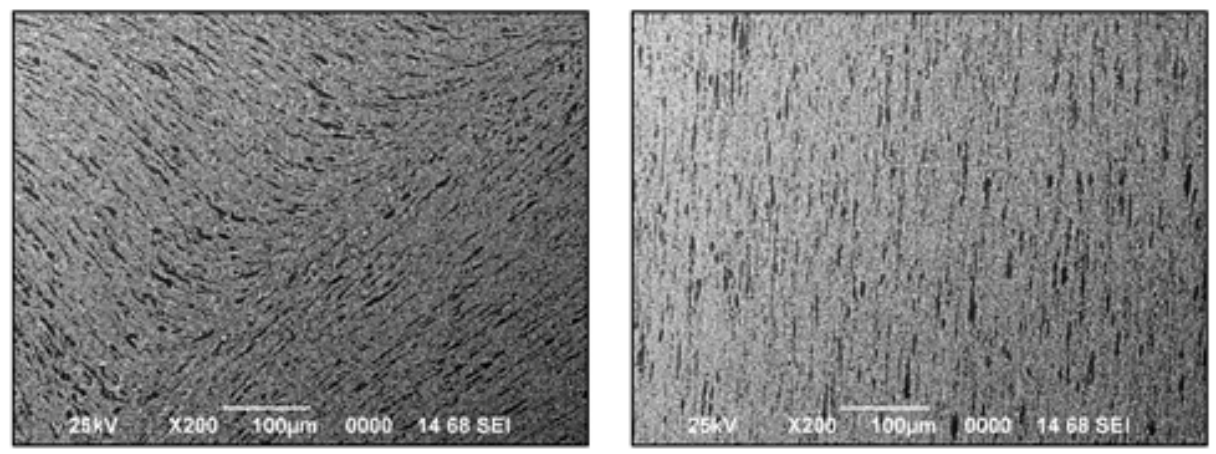

Figure 5: Typical microstructures of headed Ti 6-22-22 bolts in the head (left) and shank (right) regions.
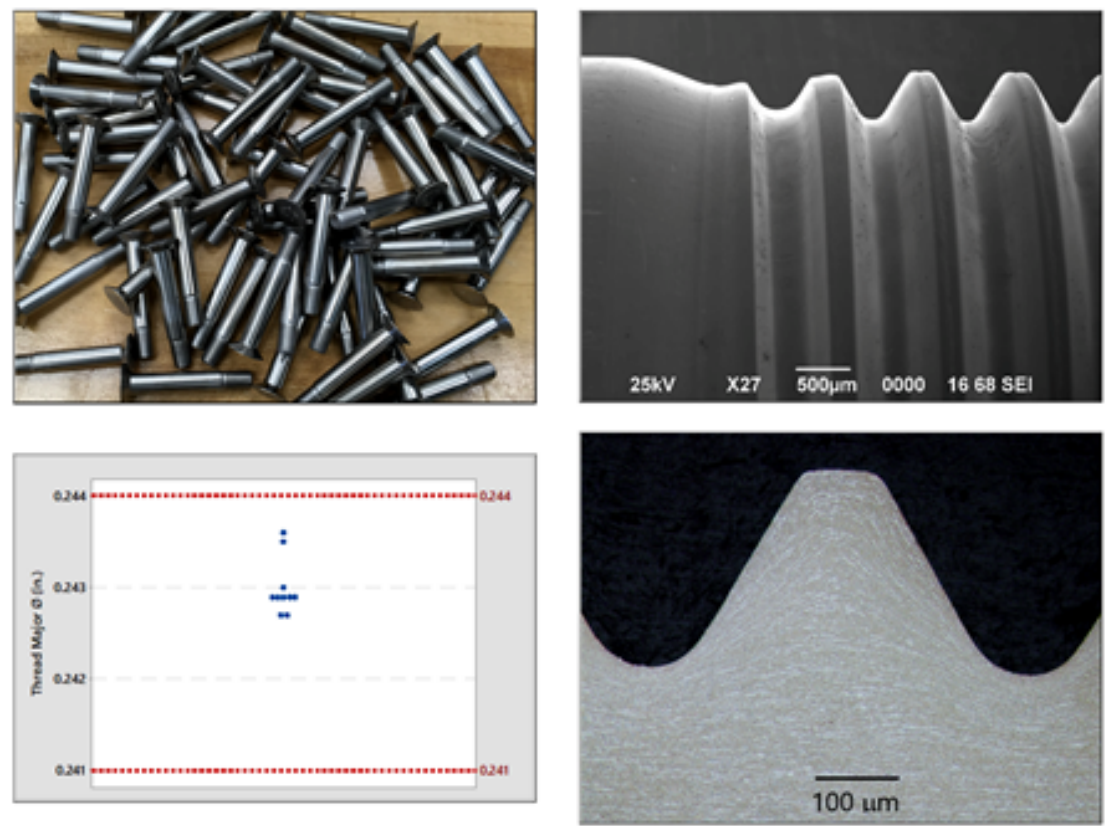

Figure 6: Finished Ti 6-22-22 fasteners along with dimensional and metallurgical characteristics in the thread section.
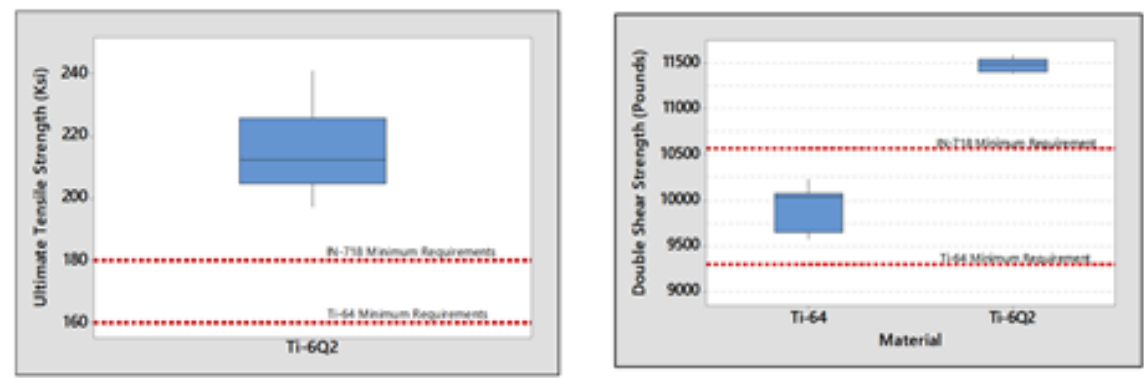

Figure 7: Tensile and double shear strength of Ti 6-22-22 (Ti-6Q2) fasteners compared with Ti 6-4 and IN-718.

\section{$\underline{\text { Acknowledgements }}$}

The authors acknowledge significant contributions by various members from Arconic and support by Carpenter Dynamet.

\section{$\underline{\text { References }}$}

1. J.G. Ferrero and S. S. Sweet, "Evaluation of The Relationship Between Tensile and Double Shear Strength for Various Titanium Alloys", Proceedings of the 13th World Conference on Titanium, TMS, https://doi.org/10.1002/9781119296126.ch165 (2016) 965-970.

2. S. R. Seagle, G.S. Hall, and H.B. Bomberger, "Development of a Deep-Hardenable Alloy for Intermediate-Temperature Applications", Titanium Science and Technology, Plenum Press, NY (1973) 1981-1991. 
3. H.R. Phelps and J.R. Wood, "Correlations of Mechanical Properties and Microstructures of Ti-6Al-2Sn-2Zr-2Mo-2Cr0.25Si Titanium Alloy," in Titanium '92; Science and Technology, edited by F.H. Froes, I.L. Caplan, Volume I, (1992) 193200.

4. G. Lutjering and J.C. Williams, Titanium, 2nd ed., Springer-Verlag, New York (2007). 